\title{
Funcionalidade comunicativa em indivíduos acometidos com esquizofrenia por meio da intervenção fonoaudiológica
}

Communicative functionality in individuals with schizophrenia

Funcionalidad comunicativa en individuos con esquizofrenia

Tatiana Moura dos Santos Andrade ORCID: https://orcid.org/0000-0001-7281-6226

Faculdade de Ensino Superior do Piauí, Brasil E-mail: tatianamourathe@gmail.com

Gislene Mariana Pereira Castelo Branco ORCID: https:/orcid.org/0000-0003-4393-771X Faculdade de Ensino Superior do Piauí, Brasil E-mail:gi.marianna@hotmail.com

Ruth Raquel Soares de Farias

ORCID: https://orcid.org/0000-0002-0988-0900 Faculdade de Ensino Superior do Piauí, Brasil E-mail: ruthraquelsf@gmail.com

\begin{abstract}
Resumo
É de suma importância discutir sobre a saúde mental, explorando mais informações e assim conscientizando sobre as diversas formas de tratamento que essas pessoas podem adquirir. Dessa forma, o presente artigo aborda uma discussão sobre a saúde mental, precisamente sobre a Esquizofrenia e sua funcionalidade comunicativa em pacientes diagnosticados com a doença. O objetivo principal desta pesquisa foi investigar as dificuldades na comunicação e habilidades linguísticas, que podem afetar o relacionamento interpessoal de indivíduos com diagnóstico de esquizofrenia. A metodologia utilizada para melhores investigações de dados para este trabalho foi pesquisa bibliográfica integrativa que permite uma melhor fundamentação teórica e levantamento de dados verídicos e críticos. Constatou-se na realização de tarefas por pessoas com esquizofrenia todas apresentam alterações, sendo os aspectos mais prejudicados a questões atinentes ao discurso e a pragmática, que não devem ser relacionados somente aos aspectos linguísticos, mas também às características de alteração do pensamento e da cognição, ao embotamento afetivo e as questões sociais referentes a esse transtorno.
\end{abstract}

Palavras-chave: Saúde mental; Comunicação; Fonoaudiologia.

\begin{abstract}
It is extremely important to discuss mental health, exploring more information and thus raising awareness about the different forms of treatment that these people can acquire. Thus, this article addresses a discussion about mental health, precisely about Schizophrenia and its communicative functionality in patients diagnosed with the disease. The main objective of this research was to investigate the difficulties in communication and language skills, which can affect the interpersonal relationships of individuals diagnosed with schizophrenia. The methodology used for better data investigations for this work was an integrative bibliographic research that allows a better theoretical foundation and a survey of true and critical data. It was found in the performance of tasks by people with schizophrenia, all of them present alterations, with the aspects most affected by issues related to discourse and pragmatics, which should not only be related to linguistic aspects, but also to the characteristics of altered thinking and cognition, affective blunting and social issues related to this disorder.
\end{abstract}

Keywords: Mental health; Communication; Speech therapy.

\section{Resumen}

Es sumamente importante hablar de salud mental, explorar más información y así concienciar sobre las diferentes formas de tratamiento que pueden adquirir estas personas. Así, este artículo aborda una discusión sobre salud mental, precisamente sobre la esquizofrenia y su funcionalidad comunicativa en pacientes diagnosticados con la enfermedad. El objetivo principal de esta investigación fue investigar las dificultades en las habilidades comunicativas y del lenguaje, que pueden afectar las relaciones interpersonales de las personas diagnosticadas con esquizofrenia. La metodología utilizada para mejores investigaciones de datos para este trabajo fue una investigación bibliográfica integradora que permite una mejor base teórica y un levantamiento de datos verdaderos y críticos. Se encontró en el desempeño de tareas por parte de personas con esquizofrenia, todas ellas presentan alteraciones, siendo los aspectos más afectados por cuestiones relacionadas con el discurso y la pragmática, que no solo deben estar relacionadas con 
los aspectos lingüísticos, sino también con las características del pensamiento alterado. y cognición, embotamiento afectivo y problemas sociales relacionados con este trastorno.

Palabras clave: Salud mental; Comunicación; Terapia del lenguaje.

\section{Introdução}

A esquizofrenia é um assunto pouco explorado em relação a comunicação atrelada à fonoaudiologia, este fato chama a atenção por ser relevante quando se trata de expressão comunicativa, na linguagem de indivíduos esquizofrênicos. Apesar de essa temática ser abordada de maneira limitada nos estudos fonoaudiológicos, a grande maioria dos pacientes com esquizofrenia têm dificuldades em concatenar espaço e tempo nos seus atos comunicativos. Sendo assim, são fatores que estão associados às características de diversas patologias de indivíduos inseridos na nossa sociedade (Santos, 2017).

As características mais comuns de pessoas acometidas pela esquizofrenia estão relacionadas a uma serie de sintomas, ressaltando-se delírios; alucinações; pensamentos confusos; indiferença afetiva; discurso desorganizado; comportamento motor estereotipado, com tiques ou catatônico; dificuldade em manter a concentração; interesse excessivo por religião ou ocultismo e risco de suicídio ou de ferir gravemente outras pessoas. Assim como todo transtorno psicológico, o diagnóstico é baseado no DSM-V, a partir da análise de sintomas, já que, além de a doença ter traços em comum com outros distúrbios, existem diferentes tipos de esquizofrenia. Também é importante observar a presença de demais sintomas que a acompanham, a exemplo de depressão, ansiedade, TOC e fobia social (Picolotto, 2020).

Em conformidade com Santos et al., (2014), uma das alterações constatadas na esquizofrenia é a dificuldade da comunicação que está diretamente ligada a algumas patologias. Por esse motivo, a saúde mental do indivíduo esquizofrênico pode ficar comprometida, entre transtorno mental e alterações nas habilidades cognitivas, fazendo com que a comunicação linguística chegue a ficar inapropriada, a fala desarticulada, incoerente ou vaga, levando a um quadro psicótico alterado.

Muitas das vezes esse indivíduo não esteja se comunicando, porque ouve pobreza no conteúdo da fala, esse quadro de comprometimento da comunicação do sujeito é amplo e pode afetar o processamento de aquisição comunicativo como discursivo, pragmático, léxico-semântico e prosódico, que por sua vez, podem interferir na expressão da linguagem e na comunicação geral devido ao quadro da esquizofrenia. Dentre outras dificuldades psíquicas está o isolamento social, que afeta também a compreensão de discurso, as perturbações de linguagem, postura social, gerando alucinações e delírios, além de comportamentos inadequados de modo geral (Almeida, Cunha \& Souza, 2013).

A intervenção fonoaudiológica permite melhorias da comunicação, do humor e da expressão corporal e amplia autonomia dos participantes, demonstrada pelo desejo de voltar às atividades cotidianas e em sociedade. Por meio de sessões de fonoaudiologia, as quais podem ser utilizadas como uma nova terapia em prol da reabilitação de pessoas com esquizofrenia, facilitam o retorno aos ambientes sociais. A intervenção fonoaudiológica proporciona melhorias do comportamento comunicativo de indivíduos com esquizofrenia e por meio da ampliação de sua atuação, ao utilizar a comunicação como instrumento de socialização contribui para a melhoria das condições de vida dessas das pessoas em sofrimento mental (Picolotto, 2020).

A partir desse pressuposto, surge o seguinte questionamento: Como a comunicação pode ser importante no processo das dificuldades interpessoais linguísticas e cognitiva no tratamento de indivíduos acometidos pela esquizofrenia?

Considerando esses aspectos, esta pesquisa tem por objetivo geral investigar as dificuldades na comunicação e habilidades linguísticas, que podem afetar o relacionamento interpessoal de indivíduos com diagnóstico de esquizofrenia. A fim de alcançar esse propósito, foi elencado os seguintes objetivos específicos: Analisar o papel do fonoaudiólogo do tratamento de indivíduos com diagnóstico de esquizofrenia; Compreender como a intervenção fonoaudiológicas trabalha as habilidades sociais e cognitivas para organizar o diálogo desse indivíduo; Descrever as principais estratégias para aprimorar a comunicação e habilidades linguísticas, por meio dos resultados encontrados nos trabalhos já concluídos. 
Assim, a pesquisa é bastante pertinente, podendo servir como mais uma fonte de pesquisa para atuais e futuros acadêmicos do curso de fonoaudiologia, em especial, aqueles, que procuram entender as consequências da má utilização da voz humana.

\section{Referencial Teórico}

\subsection{Importância da comunicação}

A comunicação é uma necessidade imprescindível para qualquer pessoa, pois permite a aquisição de conhecimentos e experiências, ajudando as pessoas a se manterem ativas na vida social e familiar. Quando a comunicação é prejudicada, pode causar frustração pessoal levando o indivíduo ao auto isolamento e à depressão (Picolotto, 2020).

A comunicação não verbal está sempre presente, seja ela implícita ou explicitamente. O importante neste tipo de linguagem são os signos comunicacionais, que atuam através da ordem do não dito e se constituem como formas de linguagem não verbais altamente eficientes no mundo da comunicação humana. Este tipo de comunicação é de extrema importância, pois indivíduos com esquizofrenia apresentam distúrbios de fala e linguagem que impactam fortemente sua integração à sociedade. O não verbal trata-se de uma linguagem comunicativa um tanto poderosa, pois a mesma possui capacidade de influenciar tanto de forma positiva ou negativamente um discurso. A linguagem não verbal pode ser reveladora das relações de comunicação, expõe verdades, reforça ideias, favorece ou dificulta o entendimento, dá ênfase à comunicação. A linguagem não verbal pode estar expressa nos sons, nos gestos, nas expressões faciais, na motricidade corpórea, na arte e nos símbolos com significação conotativa (Santos \& Silva, 2016).

De acordo com Santos (2017), existem casos que essas dificuldades são denominadas de "desordens cognitivolinguísticas", justamente pelo fato de afetar as diferentes modalidades da linguagem e os seus principais componentes, podendo ser suas causas, adquiridas, ambientais ou evolutivas. A partir disso, compreende-se que esses distúrbios abrangem dificuldades no que diz respeito às entonações emocionais, tanto na compreensão como na execução.

Segundo Cunha (2009) a partir da interlocução entre os saberes e práticas em fonoaudiologia, psiquismo e saúde pública, a comunicação exerce um papel importante de interface nestas áreas. A relação entre linguagem e psiquismo pode ser biológica, a partir da relação cérebro-linguagem, comportamental, em termos de aprendizagem e desenvolvimento, e, psicodinâmica, com a indissociabilidade entre os funcionamentos da linguagem e psíquico. Nesta pesquisa, o prisma a ser adotado entende que alterações orgânicas, psíquicas e/ou cognitivas podem interferir na linguagem e comunicação, podendo comprometer seriamente as relações sociais e causando exclusão (Gonçalves, Tochetto \& Primo, 2015).

\subsection{Esquizofrenia}

A esquizofrenia é um distúrbio cerebral desafiador que muitas vezes torna difícil distinguir entre o que é real e irreal, pensar com clareza, controlar as emoções, relacionar-se com os outros e funcionar normalmente. Afeta a maneira como uma pessoa se comporta, pensa e vê o mundo, podendo progredir em diversas fases, embora a duração e os padrões dessas fases possam variar. Pacientes esquizofrênicos tendem a apresentar sintomas psicóticos em média de 12 a 24 meses antes de procurarem atendimento médico, mas o transtorno agora é reconhecido mais cedo durante seu curso (Almeida, Cunha \& Souza, 2014).

Dessa maneira, doença foi conceitualizada desde o século XIX, de acordo com Silva (2006), para Emil Kraepelin a esquizofrenia é identificada através do diagnóstico de demência precoce, o mesmo autor também afirma que Eugen Bleuler tornou-se um cientista de grande relevância ao incluir na caracterização da esquizofrenia, o termo de comportamento advindo de alucinações e delírios. Em conformidade, Santos et al., (2014), alegam que a causa exata da esquizofrenia não se sabe, o que pode levar a essa doença, ou distúrbios de pensamentos, delírios e alucinações mas existem fatores que levam a essa 
combinação de características relevante, como genética, ambiente, alterações cerebrais, de fato que, modifica e influencia as condições desse indivíduo. Neste viés, Santos et al., (2014), diz que esse distúrbio aparece quase sempre na adolescência podendo persistir a vida inteira, o tratamento pode ajudar de forma positiva nessas alterações de comportamentos desorganizados e comunicação restrita, fazendo com que as atividades cotidianas fiquem de forma normal por algum período de tempo.

Além disso, as dificuldades de comunicação estão associadas a diversas patologias, entretanto, poucos são os dados epidemiológicos sobre essas alterações na área da saúde mental. Ressalta-se também que a esquizofrenia era dividida em quatro tipos, mas de acordo com critério da DSM-V está na categoria geral e os sintomas mais comuns são delírios e alucinações (Almeida, Cunha \& Souza, 2014).

A Lei 10.216 assegura aos indivíduos que forem diagnosticados com transtorno mental, direitos como: ser tratado com respeito e humanidade, visando alcançar sua recuperação pela inserção na família, na comunidade e no trabalho, Acesso ao melhor tratamento do sistema de saúde. Com isso, a legislação visa proporcionar à pessoa com diagnóstico de esquizofrenia ter benefícios que propiciem uma qualidade vida, ser protegido contra qualquer forma de exploração e abuso, ter direito à presença médica, a qualquer momento, para esclarecer a necessidade ou não de sua hospitalização involuntária, receber o maior número de informações a respeito de sua doença e de seu tratamento, ter livre acesso aos meios de comunicação disponíveis, ser tratado, preferencialmente, em serviços comunitários de saúde mental, ser tratado em ambiente terapêutico pelos meios menos invasivos possíveis (Brasil, 2001).

Com isso, a legislação propõe a pessoa com diagnóstico de esquizofrenia ter benefícios que possam propiciar qualidade de vida, acesso a melhores tratamentos, atendimento médico e conscientização sobre seu quadro de diagnóstico. Outro ponto a ser destacado, é que a esquizofrenia é uma doença crônica que pode progredir em diversas fases, embora a duração e os padrões dessas fases possam variar. Pacientes esquizofrênicos tendem a apresentar sintomas psicóticos em média de 12 a 24 meses antes de procurarem atendimento médico, mas o transtorno agora é reconhecido mais cedo durante seu curso, por isso é de grande importância ter assistências legislativas.

Evidenciam-se também, de acordo com Santos et al., (2014), que a esquizofrenia é um transtorno mental crônico generalizado de etiologia multifatorial e prevalência global de $0,3 \%$ a $0,7 \%$. Salienta-se que a forma mais comum é a esquizofrenia paranoide, ou esquizofrenia com paranoia, como é frequentemente chamada, pessoas com esquizofrenia paranoide têm uma percepção alterada da realidade e podem ver ou ouvir coisas que não existem, falar de maneiras confusas, acreditar que outros estão tentando prejudicá-los ou sentir que estão sendo constantemente observados, dessa forma, causando de problemas de relacionamento, que podem interromper as atividades diárias normais como tomar banho, comer ou fazer recados e levar ao abuso de álcool e drogas na tentativa de automedicação.

Para Silva (2006), a esquizofrenia na maioria das vezes é diagnosticada em jovens e adultos, dessa maneira, a prevalência relatada da esquizofrenia é variável, uma vez que diversos estudos têm usado diferentes métodos de determinação. O mesmo autor pontua também que Kütner, Martín e Almenara publicaram um estudo de revisão crítica a respeito das variações de prevalência na esquizofrenia, eles revisaram 53 estudos epidemiológicos que relataram 76 taxas de prevalência, oscilando a prevalência de esquizofrenia em 1 e 45 para cada 1.000 habitantes.

Santos et al., (2014), relatam que a idade de início da doença é maior em homens, enquanto as definições que permitem incluir mais sintomas de humor e fatos breves mostram risco equivalente para ambos os sexos. Sob o mesmo ponto de vista, os autores descrevem que o aparecimento dos sinais ocorre geralmente entre os 15 e 45 anos de idade e com maior frequência no início da juventude (15-25 anos).

Em relação ao que de fato leva uma pessoa a ter a esquizofrenia, Aguiar-Valles (2011) e Rocha (2007) declara que as causas da esquizofrenia ainda não foram desvendadas, porém há uma anuência em relacionar as alterações presentes no quadro 
do indivíduo esquizofrênico com componentes genéticos e biopsicossociais. Além do que as alterações anatômicas e fisiológicas cerebrais e a influência de fatores pré e perinatais também podem influenciar no desenvolvimento do transtorno.

Ramos e Bortagarai (2012), alegam que os sintomas da esquizofrenia são divididos em duas categorias: positivos e negativos. Os sintomas positivos incluem distúrbios de percepção (alucinações), linguagem (linguagem desorganizada), organização comportamental (comportamento gravemente desorganizado ou catatônico) e exageros do pensamento inferencial (delírios). Entretanto, apesar desses sintomas estarem divididos, Rocha (2007) diz que qualquer um desses sintomas, se observado isoladamente, não assegura a presença do transtorno.

Em vista disso, Tenório (2016), fala que os sintomas positivos incluem duas dimensões que podem estar relacionadas a diferentes mecanismos neurais subjacentes e correlações clínicas, a saber: a dimensão psicótica, que inclui delírios e alucinações, e a dimensão desorganizada, que inclui comportamento e fala desorganizado.

Por outro lado, os sintomas negativos são caracterizados por uma diminuição ou perda de emoções que incluem percepção, pensamento inferencial, linguagem e comunicação, organização comportamental, afetividade, fala, capacidade hedônica, vontade, motivação e atenção. Almeida (2019), que desses sintomas, podem ser notadas as restrições na área de intensidade de expressão emocional (achatamento afetivo), fluência e produtividade de pensamento e linguagem (alogia) e o início de comportamento direcionado a um objetivo (abulia).

Segundo Rocha (2007), os indivíduos com predomínio dos sintomas negativos têm menos aquisições acadêmicas, menor sucesso na obtenção de emprego, menor desempenho em tarefas cognitivas, ou seja, caracterizando pior prognóstico do que aqueles que apresentam maior sintomatologia dos sintomas positivos. Assim, as perturbações no processo de socialização do indivíduo com diagnóstico de esquizofrenia estão mais destacadas na sintomatologia negativa da doença e, considerando-se que a comunicação é resultado de um processo de socialização do ser humano, pode-se prever que ela estará mais prejudicada nos indivíduos com manifestação desses sintomas.

Há diversas modalidades terapêuticas disponíveis no tratamento dos indivíduos com diagnóstico de esquizofrenia. Para Dalgalarrondo (2000) e Kaplan (1997), definir o melhor tratamento, é importante considerar que o planejamento terapêutico deve ser moldado segundo as necessidades específicas de cada pessoa. Além disso, Kaplan (1997) fala que a esquizofrenia, por ser um transtorno mental complexo, exige um tratamento completo, que apresenta enfoque terapêtico nas diversas vertentes existentes, sendo os principais os tratamentos somáticos e os psicossociais. Assim, como agentes farmacológicos, devem ser usadas para tratar possíveis alterações químicas ocasionadas pelo transtorno, estratégias não farmacológicas devem abordar questões não biológicas.

Mediante as informações supracitadas o profissional fonoaudiólogo deve se dedicar ao estudo dos processos normais e patológicos da comunicação humana, para que ele possa adquirir sua especialização voltada para tratamento de distúrbios tanto de linguagem como de comunicação na esquizofrenia, a fim de que, possa estar colaborando para a melhoria da qualidade de vida do sujeito, pois a comunicação é essencial para o desenvolvimento de habilidades pessoais e para a integração social e profissional (Santos \& Silva, 2016).

É oportuno analisar as evidências científicas sobre a atuação fonoaudiológica na esquizofrenia, fato que oferece dados tanto para o atendimento clínico quanto para pesquisas futuras. Assim, o objetivo deste estudo foi sintetizar as evidências existentes na literatura a respeito da Fonoaudiologia, como parte dos currículos de reabilitação de pacientes com esquizofrenia, que enfatizam as habilidades de fala e linguagem avaliadas, configurações de terapia e abordagem terapêutica.

\subsection{Intervenção da fonoaudiologia no desenvolvimento de pessoas com esquizofrenia}

A Fonoaudiologia é uma ciência nova que suscita a cada dia novos conhecimentos nos quais devemos ter uma parcela de colaboração no desenvolvimento da ciência e, até certo ponto, a integração e o bem-estar do ser humano dependem da 
comunicação. Neste modo, Oliveira (2011) diz que a grande importância à presença do profissional fonoaudiólogo na recuperação, aprimoramento e reintegração do homem à sociedade.

Santos e Silva (2016, p.1448), afirmam que: "O fonoaudiólogo é o profissional especializado na comunicação humana que, por surgir com uma relação próxima à Medicina e à Educação, teve em sua trajetória uma prática preocupada primordialmente com a reabilitação". Nesse sentido, pode-se entender que este profissional trabalha com diversos aspectos da comunicação, aliado a outras áreas, na busca de resultados satisfatórios para seus pacientes. Corroborando com isso, Almeida, Cunha e Souza (2014) ressaltam que o fonoaudiólogo é o profissional competente a construir atividades terapêuticas que resgatem a comunicação, por meio de trocas afetivo-simbólicas, de vivências dialógicas, conversacionais".

Para compreender do papel do fonoaudiólogo na saúde mental, é necessário refletir sobre a construção histórica psiquiátrica no Brasil. Assim, Almeida, Cunha e Souza (2014) norteiam o entendimento acerca desse aspecto evidenciando que o processo de Reforma Psiquiátrica no Brasil ocorreu em 1970, com propósito de superação na busca de uma rede humanizada de atenção à saúde mental - substitutiva ao modelo centrado na internação hospitalar - e a fiscalização e redução progressiva.

Neste termo, novas alternativas estão sendo construídas visando contribuir para a formação de um novo espaço social para o indivíduo com transtornos mentais. Mediante esse contexto de desinstitucionalização inicia-se a construção de em uma nova ótica no cuidado em saúde mental, a fonoaudiologia cresce no momento em que há um aumento do trabalho das equipes multi e interdisciplinares e no desafio de mudança deste paradigma (Santos \& Silva, 2016).

A partir daí a Reforma Psiquiátrica se consolida como política pública, e a Fonoaudiologia brasileira passa atuar de forma mais efetivamente nas instituições psiquiátricas a partir de 1992, a partir da criação da Portaria 224/92, também elaborada pelo Ministério da Saúde, na qual uma das diretrizes é a multiprofissionalidade na prestação de serviços aos doentes mentais (Borges, 2012).

Com base nessa perspectiva, a fonoaudiologia em um processo multiprofissional passa a ter espaço, constituindo por atuação em equipe, caracterizada por (Peduzzi, 2001) como uma modalidade de trabalho coletivo e de reciprocidade entre as múltiplas intervenções técnicas e a interação dos diferentes profissionais. Mais do que isso, na medida em que se delineia um novo cenário na Saúde Mental, que parte de uma ética do não isolamento e da construção de alternativas para promover a convivência e a circulação.

Diante disso, observa-se que ainda há carência de pesquisas nesta área da Fonoaudiologia na Esquizofrenia, uma vez que foram encontrados para fundamentação deste trabalho poucos registros relacionados à temática. Logo, isso demonstra a grande necessidade de uma intervenção fonoaudiológica maior para que os usuários alcancem uma melhor qualidade de vida, tendo em vista, uma interação comunicativa mais adequada e eficiente. Portanto, as informações obtidas servem de base para a sugestão de que há necessidade urgente de estudos com maior homogeneidade metodológica e que contenham procedimentos mais relacionados à intervenção terapêutica.

\section{Metodologia da Pesquisa}

O método utilizado nesta pesquisa foi uma revisão bibliográfica integrativa, em que teve como finalidade sintetizar os conhecimentos sobre a temática de maneira organizada para uma melhor compreensão da análise do estudo. Segundo Souza, Silva e Carvalho (2010, p. 103), a revisão integrativa: "é a mais ampla abordagem metodológica referente às revisões, permitindo a inclusão de estudos experimentais e não experimentais para uma compreensão completa do fenômeno analisado".

O levantamento da pesquisa bibliográfica foi realizado pela internet, por meio de bases de dados e plataformas digitais, como SCIELO, CAPES e PUBMED, em busca de estudos atendam a temática da pesquisa, contribuindo assim para uma melhor execução do estudo. Cruzando os seguintes descritores: Comunicação, esquizofrenia e fonoaudiologia. Utilizando o operador booleano "AND". Estratégia de busca utilizada na SCIELO, "Comunicação AND esquizofrenia AND 
fonoaudiologia", na CAPES “Comunicação AND esquizofrenia AND fonoaudiologia”, e PubMed "Communication AND schizophrenia AND speech therapy".

Foram incluídos os artigos científicos completos e disponíveis gratuitamente, nos idiomas português e inglês, publicados entre os anos de 2012 a 2021. Sendo excluídos os artigos que não atenderam a temática, os duplicados, os que estavam fora do recorte temporal e produções de literatura cinzenta (dissertações e teses).

Como base nas pesquisas selecionadas buscou-se compreender conceitos e a intervenção da Fonoaudiologia com pessoas com esquizofrenia. Tendo em vista que, o fundamento teórico foi estruturado para oferecer uma melhor compreensão sobre a funcionalidade comunicativa nesses indivíduos frente aos impactos psicossociais.

A Figura 1 consiste no quantitativo de artigos que foram utilizados na pesquisa, assim como a base de dados na qual estão disponibilizados, demonstrando ainda o número de artigos que foram excluídos.

Figura 1: Quantidade de artigos conforme a base de dados.

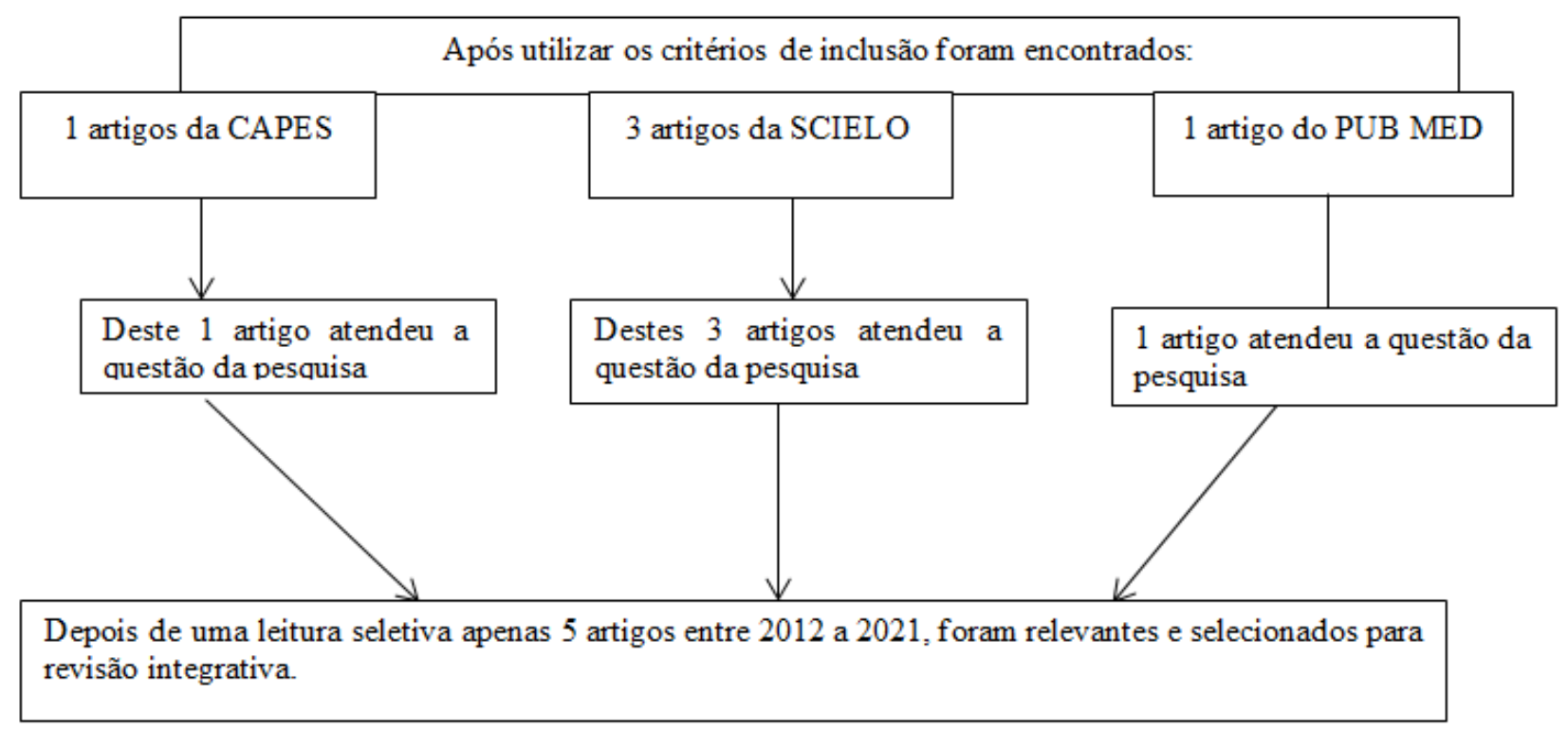

Fonte: Autores (2021).

Após a análise dos artigos que foram incluídos na pesquisa realizou-se a elaboração do quadro 1, o qual apresenta o autor, o título da pesquisa, a metodologia que foi empregada e os resultados que foram conseguidos com a intervenção da Fonoaudiologia em pessoas com esquizofrenia.

\section{Resultados e Discussão}

O Quadro 1 discrimina, conforme a pesquisa bibliográfica integrativa, a importância da intervenção Fonoaudiológica em pessoas com esquizofrenia. 
Quadro 1. Artigos sobre os benefícios da intervenção da fonoaudiologia para esquizofrênicos.

\begin{tabular}{|c|c|c|c|}
\hline Autor/Título & $\begin{array}{c}\text { Base de } \\
\text { dados }\end{array}$ & Metodologia & Resultados \\
\hline $\begin{array}{lr}\text { Santos (2017) } \\
\\
\text { A efetividade da } \\
\text { intervenção } \\
\text { fonoaudiológica } \\
\text { grupal } \\
\text { comportamento } \\
\text { comunicativo } \\
\text { indivíduos de } \\
\text { diagnóstico com } \\
\text { esquizofrenia }\end{array}$ & SCIELO & $\begin{array}{l}\text { Trata-se de um estudo quase experimental, } \\
\text { quantitativo analítico-exploratório. Foram } \\
\text { incluídos usuários de um Centro de Atenção } \\
\text { Psicossocial III (CAPS III) com diagnóstico } \\
\text { de esquizofrenia, divididos em } 2 \text { grupos: } \\
\text { Grupo Experimental (GE), compondo o } \\
\text { Grupo de Intervenção Fonoaudiológica } \\
\text { (GIF) e Grupo Controle (GC). O } \\
\text { comportamento comunicativo foi avaliado } \\
\text { através da Bateria MAC Breve. O GIF foi } \\
\text { realizado em } 2 \text { sessões semanais, totalizando } \\
24 \text { sessões. Após esse período, os indivíduos } \\
\text { foram reavaliados. A análise ocorreu por } \\
\text { meio dos Testes não paramétricos de Mann } \\
\text { Whitney e o Teste de Correlação de Pearson. }\end{array}$ & $\begin{array}{l}\text { Participaram } 19 \text { indivíduos, de ambos os sexos, com idade entre } 19 \\
\text { e } 59 \text { anos, escolaridade mínima de } 5 \text { anos, sendo que } 14 \\
\text { participaram do GE e } 5 \text { do GC. No GE, foi possível observar que } \\
\text { houve melhora no comportamento comunicativo após a } \\
\text { intervenção fonoaudiológica em todas as tarefas avaliadas, exceto } \\
\text { na tarefa de Escrita. Já no GC, não foram observadas alterações } \\
\text { significativas comparando a avaliação e a reavaliação após } 12 \\
\text { semanas. }\end{array}$ \\
\hline $\begin{array}{l}\text { Almeida, Cunha e } \\
\text { Souza (2014) } \\
\text { Características e } \\
\text { demandas } \\
\text { fonoaudiológicas de } \\
\text { pacientes adultos } \\
\text { portadores de } \\
\text { transtornos mentais } \\
\text { (esquizofrenia) e } \\
\text { institucionalizados } \\
\text { em um Centro de } \\
\text { Atenção Integral à } \\
\text { Saúde de São Paulo }\end{array}$ & SCIELO & $\begin{array}{l}\text { Trata-se de estudo retrospectivo, de natureza } \\
\text { quantitativa e descritiva, que analisou } \\
\text { prontuários de } 106 \text { sujeitos, de ambos os } \\
\text { sexos, com idade média de } 62 \text { anos, } \\
\text { atendidos individualmente no Setor de } \\
\text { Fonoaudiologia do CAIS-SR. A análise dos } \\
\text { dados foi realizada por meio de estatística } \\
\text { descritiva. }\end{array}$ & $\begin{array}{l}\text { Os resultados mostram que os moradores encaminhados ao setor } \\
\text { de fonoaudiologia (25\% do total de moradores do CAIS) são, em } \\
\text { sua maioria, mais idosos e estão asilados há mais tempo. A maioria } \\
\text { dos sujeitos que receberam atendimento individual em } \\
\text { Fonoaudiologia }(58,5 \%) \text { foi atendida na especialidade Linguagem. } \\
\text { A segunda categoria mais frequente foi Motricidade } \\
\text { orofacial+Linguagem }(30,2 \%) \text {. Vinte e dois (20,8\%) dos } \\
\text { indivíduos que receberam atendimento individual apresentaram } \\
\text { alterações de comunicação em função também de problemas } \\
\text { auditivos. Sendo assim, a maioria das queixas e atendimentos } \\
\text { fonoaudiológicos circulou em torno de alterações de comunicação } \\
\text { e linguagem dos moradores atendidos na instituição. }\end{array}$ \\
\hline $\begin{array}{lr}\text { Santos (2017) } & \\
\text { Comportamento } & \\
\text { comunicativo } & \text { de } \\
\text { indivíduos } & \text { com } \\
\text { diagnóstico } & \text { de } \\
\text { esquizofrenia: } & \\
\text { efetividade } & \text { da } \\
\text { intervenção } & \\
\text { fonoaudióloga } & \\
\end{array}$ & SCIELO & $\begin{array}{l}19 \text { pessoas participaram do estudo e foram } \\
\text { divididas em dois grupos: Grupo } \\
\text { experimental, que passaram por uma } \\
\text { intervenção fonoaudiológica; e o Grupo } \\
\text { controle, que não passou por nenhuma } \\
\text { sessão fonoaudiológica. }\end{array}$ & $\begin{array}{l}\text { Através da fonoaudiologia o paciente consegue a melhora no } \\
\text { humor e da expressão corporal, melhor autonomia, incentivo a } \\
\text { voltar às atividades cotidianas e aperfeiçoamento da comunicação. } \\
\text { Esses são os resultados da pesquisa que foi realizada com um } \\
\text { grupo de pessoas que sofrem com esquizofrenia e passaram por } \\
\text { sessões de fonoaudiologia. Os resultados positivos foram } \\
\text { percebidos no grupo que passou por } 24 \text { sessões de fonoaudiologia } \\
\text { durante } 12 \text { semanas e nesse período participaram de várias } \\
\text { atividades. E os resultados são ótimos, já que essas sessões podem } \\
\text { ajudar os pacientes a retornar aos ambientes sociais. }\end{array}$ \\
\hline $\begin{array}{l}\text { Joyal et al., (2016) } \\
\text { Speech and } \\
\text { language therapies } \\
\text { to improve } \\
\text { pragmatics and } \\
\text { discourse skills in } \\
\text { patients with } \\
\text { schizophrenia. }\end{array}$ & CAPES & $\begin{array}{l}\text { Este artigo revisou } 18 \text { estudos que testaram } \\
\text { os efeitos da terapia ou treinamento da } \\
\text { linguagem em } 433 \text { adultos com diagnóstico } \\
\text { de esquizofrenia. }\end{array}$ & $\begin{array}{l}\text { Indivíduos com esquizofrenia apresentam deficiências de fala e } \\
\text { linguagem que impactam fortemente sua integração à sociedade. } \\
\text { Os resultados mostraram que } 14 \text { estudos de } 18 \text { levaram a melhorias } \\
\text { nas habilidades de linguagem e / ou fala. A maioria desses estudos } \\
\text { compreendeu habilidades discursivas pragmáticas ou expressivas, } \\
\text { sendo o único objetivo da terapia ou parte dela. As configurações } \\
\text { de terapia variam amplamente, desde terapia individual duas vezes } \\
\text { ao dia até terapia de grupo uma vez por semana. A abordagem } \\
\text { terapêutica foi principalmente o condicionamento operante. } \\
\text { Embora as evidências tendam a mostrar que certas áreas da } \\
\text { linguagem são tratáveis por meio de terapia, permanece difícil } \\
\text { estabelecer o tipo de abordagem que deve ser favorecida e } \\
\text { implementada para tratar os distúrbios de linguagem na } \\
\text { esquizofrenia. }\end{array}$ \\
\hline $\begin{array}{lr}\text { Teodoro } & (2013) \\
\text { intervenções } & \\
\text { terapêuticas } & \text { em } \\
\text { pessoas } & \text { com } \\
\text { esquizofrenia } & \end{array}$ & PUBMED & $\begin{array}{l}\text { Foram recuperados dez artigos nas bases } \\
\text { LILACS, SciELO e PePSIC, no período de } \\
2001 \text { a } 2011 \text {. A maior parte dos estudos é de } \\
\text { caráter empírico. }\end{array}$ & $\begin{array}{l}\text { As intervenções são predominantemente apresentadas pela } \\
\text { Fonoaudiologia, devido às características que a pessoa apresenta } \\
\text { referentes aos prejuízos na interação e nas habilidades de } \\
\text { comunicação. Desse modo geral, constatou-se que as intervenções } \\
\text { terapêuticas aplicadas em pessoas com SA têm efeitos positivos e } \\
\text { acarretam mudanças de comportamento e melhor qualidade de } \\
\text { vida, além de ser observado que o trabalho terapêutico associado à } \\
\text { intervenção com a família tende a garantir maiores benefícios a } \\
\text { ambos. }\end{array}$ \\
\hline
\end{tabular}

Fonte: Autores (2021).

Após análises de várias teorias, presentes, principalmente em artigos científicos, livros e consultas na internet, analisando, especialmente revistas científicas, referente à esquizofrenia chegou ao resultado de que dentre os déficits cognitivos nesse transtorno apresentam alterações a serem consideradas na avaliação neuropsicológica (Almeida et al., 2014).

Dentre as características apresentadas por pessoas com esquizofrenia ressaltam-se velocidade de processamento: 
rapidez em que são executadas tarefas simples que demandam funcionamento dos processos cognitivos; memória e aprendizagem verbal: capacidade de aprender e recordar informações verbais; memória e aprendizagem visual: habilidade de aprender e recordar informações visuais; raciocínio e resolução de problemas: capacidade de pensar/raciocinar em atividades novas utilizando conhecimentos previamente adquiridos; memória de trabalho: capacidade de retenção e manipulação de informações para utilização imediata; atenção/vigilância: habilidade de manter a atenção focada em uma atividade (Ferreira Júnior et al., 2015).

A efetividade da intervenção fonoaudiológica no comportamento comunicativo de indivíduos com diagnóstico de esquizofrenia, com base em estratégias utilizadas na prática clínica da Fonoaudiologia busca trabalhar as habilidades comunicativas. Ressalta-se que a fonoterapeuta deve considerar as necessidades de quem receberá a terapia, tanto clínicas quanto pessoais, pois são essas que farão com que o indivíduo tenha interesse em seguir no tratamento, obtendo resultados efetivos, apresentando melhora no discurso, no uso da linguagem (pragmática), no vocabulário (léxico-semântica) e, principalmente, na tarefa Prosódia Emocional - Produção, o que indica que o tratamento faz com que os participantes expressem emoções por meio de entonações emocionais. Nessa perspectiva, salienta-se que o fonoaudiólogo pode contribuir de maneira significativa para o atendimento clínico e para a formulação de programas de intervenção em saúde mental (Santos, 2017).

A atuação da Fonoaudiologia em pessoas com esquizofrenia, traz benéficos referentes às características da atenção, ressaltando-se a seletividade (capacidade de focar entre um estímulo e outros concorrentes); Sustentação (manter a atenção em uma atividade repetitiva sem perder qualidade); Divisão (focar em estímulos diferentes ao mesmo tempo); Alternância (capacidade de alternar de um estímulo a outro sucessivamente); amplitude atencional (habilidade de extensão da atenção em relação à quantidade de itens que podem ser processados). cognição social: capacidade de identificar, manipular e adequar o comportamento a um determinado contexto, a partir da detecção de informações provenientes do ambiente em questão (Santos, 2017).

As funções cognitivas podem ser definidas como as primeiras a demonstrarem déficit, mesmo antes dos sintomas psicóticos. Assim os fonoaudiólogos identificando os prejuízos nessas áreas e o que se encontra preservado é de extrema importância em prol da devida estruturação de um programa de reabilitação. A esquizofrenia é um transtorno psiquiátrico que tem início cedo na vida do indivíduo e apresenta diversos déficits cognitivos, sendo essas dificuldades iniciadas antes dos sintomas aparecerem e sendo identificadas enquanto característica nuclear do distúrbio. Sete funções cognitivas parecem estar mais afetadas, a saber: vigilância/atenção; memória e aprendizagem verbal; memória e aprendizagem visual; raciocínio e resolução de problemas; velocidade de processamento; memória de trabalho e cognição social (Teodoro, 2013).

A Análise do Comportamento deve identificar, a partir de uma avaliação funcional, o que mantém determinado comportamento ocorrendo e, a partir disso, possíveis formas de intervenção pelos fonoaudiólogos objetivando mudanças no repertório do indivíduo com a finalidade de conferir uma melhor qualidade de vida e de funcionalidade. Essa modalidade de abordagem proporciona um olhar diferenciado por perceber cada ser humano em sua individualidade observando as contingências e, especialmente, as relações que estão envolvidas na sua maneira de comportamento em relação ao ambiente. Considera que comportamentos, por manterem relações com o ambiente, são totalmente passíveis de intervenção, de aprendizagem e de modificação (Lipay \& Almeida, 2007).

Com essa visão, utilizando-se de técnicas como modelagem, pareamento, esquemas de reforçamento, entre outras, é possível pensar em estratégias de reabilitação na esquizofrenia, realizando-se uma avaliação de cada paciente e montando um programa personalizado e adaptado às limitações daquele indivíduo, bem como considerando suas competências preservadas. Também é importante ressaltar que, além da aplicação das técnicas, a generalização dos comportamentos deve ser programada, uma vez que, por meio desta, faz-se possível à adaptação funcional do paciente em seu cotidiano, bem como o 
acompanhamento psiquiátrico.

Conforme se evidencia pela presente pesquisa bibliográfica, foram identificados diversos benefícios e contribuições dos profissionais da fonoaudiologia no serviço de Saúde Mental, com o fonoaudiólogo podendo atuar objetivando o estímulo da criatividade, da participação coletiva e da aprendizagem de forma que favoreçam condições capazes de facilitar a reinserção social da pessoa com esquizofrenia.

$\mathrm{Na}$ tarefa de Leitura constata-se que diminuem erros posteriormente a intervenção fonoaudiológica, havendo melhorias no desempenho comunicativo. Além disso, apresentaram melhorias na linguagem e/ou habilidades de fala (Joyal et al., 2016).

\section{Considerações Finais}

Conforme a presente pesquisa evidenciou-se que por meio da atuação dos fonoaudiólogos, intervindo diretamente em grupos de pessoas com esquizofrenia é efetiva, com a fonoaudiologia crescendo no momento que ocorre uma ampliação do trabalho das equipes multi e interdisciplinares e no desafio de mudança deste paradigma, utilizando-se da comunicação como um instrumento de socialização, contribuindo para uma ampla melhoria das condições de vida desses indivíduos.

Em contrapartida, é possível identificar que no Brasil são reduzidas as pesquisas em fonoaudiologia que abordam o trabalho com pessoas esquizofrênicas. Assim, é de suma importância, primeiramente, o estabelecimento científico com a necessidade do fonoaudiólogo nos diversos serviços de saúde mental através de avaliações dos aspectos de domínio fonoaudiológicos nesses serviços, como a comunicação. Dessa forma, sugere-se o investimento em outras pesquisas atinentes com a Fonoaudiologia sobre o tema, as quais possam sustentar cientificamente as intervenções dirigidas a esses indivíduos.

Por meio da presente pesquisa observou-se que são reduzidos as pesquisas referentes a atuação da Fonoaudiologia em pessoas com esquizofrenia, necessita-se, assim novas investigações. Dessa forma esta pesquisa serve como mais uma base teórica para fundamentar o tema, sendo direcionada a fonoaudiólogos que já se encontram em atividade, como também para atuais e futuros acadêmicos do curso de Fonoaudiologia. E sugerem-se mais estudos sobre a atuação da Fonoaudiologia em pessoas com esquizofrenia, e assim ocorra elaboração de estratégias para melhorar a atuação do fonoaudiólogo com esses pacientes.

\section{Referências}

Aguilar-Valles, A. (2011). Identificación de factores genéticos en la etiología de la esquizofrenia. Acta biol.Colomb., 16(3):129-38.

Almeida, B. P. B., Cunha, M. C. \& Sousa, L. A. P. (2014) Características e demandas fonoaudiológicas de pacientes adultos portadores de transtornos mentais e institucionalizados em um Centro de Atenção Integral à Saúde de São Paulo. Distúrbio Comun, 25(1): 27-33. https://revistas.pucsp.br/index.php/dic/article/view/14921.

Almeida, V. N. (2019). Atividade física em pacientes com esquizofrenia: estudo de caso. 2019. 44 f. TCC (Graduação). Curso de Fisioterapia, Universidade Federal de Santa Catarina. https://repositorio.ufsc.br/handle/123456789/202743.

Borges, R. F. (2012). Reforma psiquiátrica brasileira e atenção básica à saúde: desafios e possibilidades. 2012.212 f. Tese (doutorado) - Universidade Estadual Paulista, Faculdade de Ciências e Letras de Assis, http://hdl.handle.net/11449/105614.

Brasil. (2001). Assembleia Legislativa. Lei 10.216, De 6 De Abril De 2001. Dispõe sobre e proteção e os direitos das pessoas portadoras de transtornos mentais e redireciona e modelo assistencial em saúde mental. http://www.planalto.gov.br/ccivil_03/leis/leis_2001/110216.htm.

Cunha, M. C. (2009). Linguagem e Psiquismo: considerações fonoaudiológicas estritas. Tratado de Fonoaudiologia. (2a ed.), $414-8$.

Dalgalarrondo, P. (2000). Psicopatologia e Semiologia dos transtornos mentais. Editora Artmed, 2000.

Ferreira Junior, B. C., Barbosa, M. A., Barbosa, I. G., Hara, C. \& Rocha, F. L. (2015). Alterações cognitivas na esquizofrenia: atualização. Rev Psiquiatr Rio Gd Sul, 32(2): 57-63. https://doi.org/10.1590/S0101-81082010000200006

Gonçalves, M. S., Tochetto, T. M. \& Primo, M. T. (2015) Fonoaudiologia em saúde coletiva: prioridades detectadas pelos usuários de unidades básicas de saúde. Rev Fonoaudiol Brasil, 3(2): 1-3. 
Research, Society and Development, v. 10, n. 15, e568101523248, 2021

(CC BY 4.0) | ISSN 2525-3409 | DOI: http://dx.doi.org/10.33448/rsd-v10i15.23248

Joyal, M., Bonneau, A. \& Fecteau, S. (2016). Speech and language therapies to improve pragmatics and discourse skills in patients with schizophrenia. Psychiatry Res., 240(30): 88-95. http://dx.doi.org/10.1016/j.psychres.2016.04.010 PMid:27092861.

Kaplan, H. (1997). Compêndio de psiquiatria: ciência do comportamento e psiquiatria clínica. (7a ed.). Artmed.

Lipay, M. S. \& Almeida, E. C. (2007) A fonoaudiologia e sua inserção na saúde pública. Rev Cienc Méd., 16(1): 31-41.

Oliveira, M. F. (2011). Metodologia científica: um manual para a realização de pesquisas em Administração. Catalão Goiás, 1-73.

Peduzzi, M. (2001). Equipe multiprofissional de saúde: conceito e tipologia. Rev Saúde Pública, 35(1): 103-9. https://www.scielo.br/j/rs p/a/PM8YPvMJLQ4y49Vxj6M7yzt/?lang=pt\&format=pdf.

Picolotto, M. N. (2020). TCC e esquizofrenia: quais são os ganhos para os pacientes? Rev. Cognitivo. https://blog.cognitivo.com/tcc-e-esquizofrenia-quaissao-os-ganhos-para-os-pacientes/.

Ramos, A. P. \& Bortagarai, F. M. (2012). A comunicação não-verbal na área da saúde. Rev. CEFAC. 14(1): 164-170. http://dx.doi.org/10.1590/S151618462011005000067

Rocha, J. (2007). Análise da componente pragmática da linguagem de pessoas com esquizofrenia. 2007. 135 f. Dissertação (mestrado): Aveiro, Universidade de Aveiro, 2007. https://ria.ua.pt/bitstream/10773/4875/1/2007001348.pdf.

Santos, A. E. (2017). Comportamento comunicativo de indivíduos com diagnóstico de esquizofrenia: efetividade da intervenção fonoaudiológica. 2017. $210 \mathrm{f}$. Tese (doutorado), Escola de Enfermagem de Ribeirão Preto, Universidade de São Paulo, 2017. doi:10.11606/T.22.2018.tde-29052018-145117.

Santos, A. E., Pedrão, L. J., Zamberlan-Amorim, N. E., Carvalho, A. M. P. \& Bárbaro, A. M. (2014) Comportamento comunicativo de indivíduos com diagnósticos de esquizofrenia. Revista CEFAC, 16(4): 1283-1293. https://www.scielo.br/j/rcefac/a/PMKvDMtyDKS3Tk9pN9sDJ3 D/?lang=pt\#: :text=As\%20caracter\%C3\%ADsticas\%20de\%20altera\%C3\%A7\%C3\%B5es\%20de,at\%C3\%A9\%20mesmo\%20o\%20mutismo\%208.

Santos, T. D. \& Silva, M. A. (2016). Comunicação não verbal com profissionais da voz: o que se pesquisa na fonoaudiologia. Rev. CEFAC. 18(6): 1447-1455. https://www.scielo.br/j/rcefac/a/L4mN68mg4HvvdjYQxCP5MFN/?lang=pt.

Silva, R. C. B. (2006). Esquizofrenia: uma revisão. Psicologia USP, 17(4): 263-285. https://doi.org/10.1590/S0103-65642006000400014.

Souza, M. T., Silva, M. D. \& Carvalho, R. (2010). Revisão integrativa: o que é e como fazer. Revista Einstein, 8(1): 102-106. http://dx.doi.org/10.1590/s1679$45082010 \mathrm{rw} 1134$

Tenório, F. (2016). Psicose e esquizofrenia: efeitos das mudanças nas classificações psiquiátricas sobre a abordagem clínica e teórica das doenças mentais. História, Ciências, Saúde - Manguinhos, 23(4): 941-963. https://doi.org/10.1590/S0104-59702016005000018.

Teodoro, M. C. (2013). Intervenções terapêuticas em pessoas com esquizofrenia. Barbarói, 38(1): 6-25. 